
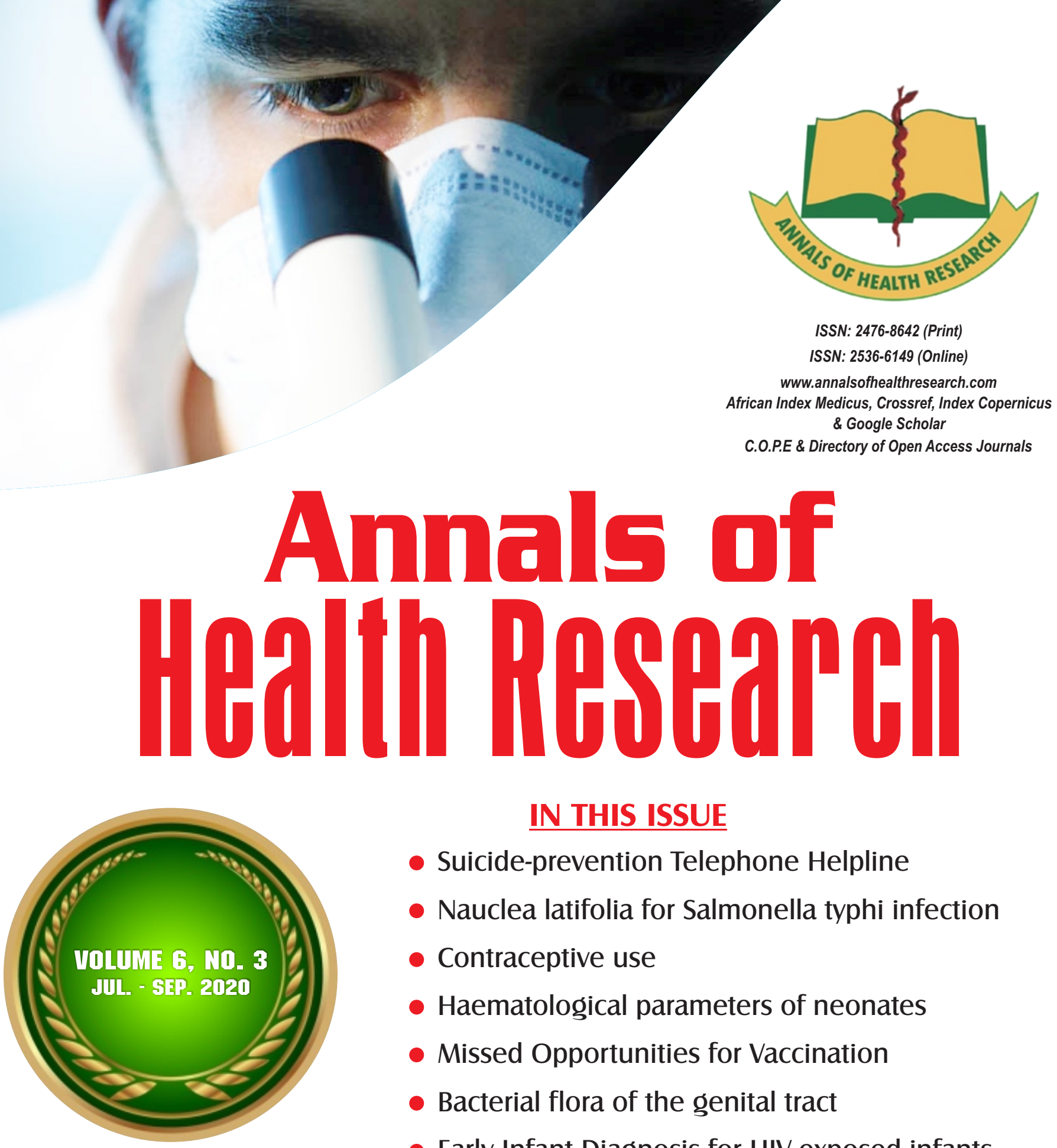

IN THIS ISSUE

- Suicide-prevention Telephone Helpline

- Nauclea latifolia for Salmonella typhi infection

- Contraceptive use

- Haematological parameters of neonates

- Missed Opportunities for Vaccination

- Bacterial flora of the genital tract

- Early Infant Diagnosis for HIV-exposed infants

- Bone markers and cardiovascular risk factors

- Attitude to termination of pregnancies

- Herpes zoster ophthalmicus

- Neonatal hyperinsulinaemic hypoglycaemia

- Paediatric perineal injury

PUBLISHED BY THE MEDICAL

AND DENTAL CONSULTANTS ASSOCIATION

OF NIGERIA, OOUTH, SAGAMU, NIGERIA.

www.mdcan.oouth.org.ng 


\title{
Factors associated with Missed Opportunities for Vaccination among children in the first year of life at a tertiary health facility in Lagos Kehinde OA*1, Kuyinu YA², Odusanya $\mathrm{OO}^{2}$
}

${ }^{1}$ Department of Paediatrics and Child Health, ${ }^{2}$ Department of Community Health and Primary Health Care, Lagos State University College of Medicine, Ikeja Lagos, Nigeria

*Correspondence: Dr OA Kehinde, Department of Paediatrics and Child Health, Lagos State University College of Medicine, 1-5 Oba Akinjobi Way, G.R.A, P.M.B 21266 Ikeja, Lagos, Nigeria.

E-mail: larakehinde93@gmail.com; ORCID - https://orcid.org/0000-0003-2568-857X.

\begin{abstract}
Background: Low vaccination rates in children remain a major problem in resource-poor areas of the world. Missed opportunities for vaccination may be one of the important contributors to the menace.

Objectives: To determine the prevalence of missed opportunities for vaccination and identify factors associated with this among Nigerian children in the first year of life.

Methods: The study was a descriptive, cross-sectional study of children who were recruited consecutively and data were obtained using a questionnaire.

Results: Two hundred and fifty-six children were recruited. Following the exclusion of 16 children with genuine contraindications to vaccination, the prevalence of missed opportunities for vaccination was $11.3 \%(27 / 240)$. Gender $(p=0.04)$ and parental socioeconomic status $(p=0.008)$ were significantly associated with missed opportunities for vaccination. Non- availability of required vaccines and reluctance to open a multi-dose vial of vaccine caused MOV in $55.5 \%$ and $51.8 \%$ of children respectively. The rate of evaluation of the vaccination status of children who were not fully vaccinated by health workers was $18.3 \%$.

Conclusion: The rate of missed opportunities for vaccination was low. Educating caregivers and encouraging health workers to evaluate the vaccination status of children at every contact with a health facility may reduce the prevalence of missed opportunities for vaccination.
\end{abstract}

Keywords: Children, Health facility, Immunization, Lagos, Missed opportunities, Vaccination.

\section{Introduction}

Vaccine-preventable diseases are important causes of morbidity and mortality among children in developing countries. ${ }^{[1]}$ It accounts for over a quarter of deaths in children less than five years. [2] The estimated number of deaths from diseases which are preventable by vaccines recommended by the World Health Organization (WHO) and diseases, for which vaccines are to be developed soon, is over 2.5 million. [3] Therefore, immunisation is one of the most important public health interventions and a cost-effective strategy to reduce both the 
morbidity and mortality associated with infectious diseases. Optimal vaccination coverage in women of childbearing age and children aged less than five years, which is one of the targets of the sustainable development goals (SDG 2), is said to occur when at least 95\% of doses appropriate for the age have been administered in that population irrespective of the age at the receipt. ${ }^{[4]}$

Many children who are partially immunized and eligible for vaccination have contacts with health facilities during post-discharge follow-up care, visits for the treatment of minor ailments, and attendance at the Well-child and Immunisation Clinics but do not receive vaccines they are due for at exit. A missed opportunity for vaccination (MOV) occurs when an eligible child visits a health facility and fails to receive all the vaccines he or she is due for. ${ }^{[4]}$ MOV among children could be attributed to the health system, health workers and caregiver factors. Health system factors may include nonavailability of vaccines ${ }^{[5]}$ as well as the practice of having fixed days for immunisation. Health workers factors may include the failure to have a practice of routine evaluation of the immunisation status of children who come in contact with the health facility for management of minor ailments, those who attend the wellchild clinics and for other purposes such as accompanying mothers attending antenatal and family planning clinics. [6] This precludes the detection of incomplete vaccination status and vaccination of eligible children at the point of exit. [7] The institution of false contraindications to immunisation which arises from the poor knowledge of the health workers, a reluctance to administer more than one vaccine to a child at a time and the fear of wastage of vaccines [4] are additional causes of missed opportunities. The lack of guardian consent for the administration of vaccines is a caregiver factor.
Studies in Nigeria gave a range of the prevalence rates of MOV of $16.9 \%$ to $39.1 \%$. [8-11] Studies from Ilorin, [8, 9] and Calabar [10] gave prevalence rates of $33.4 \%, 24.4 \%$ and $39.1 \%$ while a rate of $16.9 \%$ was recorded in Nnewi. [11] Another study in Africa gave a value of $25.7 \%$,[12] while an Argentine study reported $19.8 \%$. [13] A study from Madras, [14] in the neonatal follow up, medical outpatient and immunization clinics yielded values of $23 \%$, 35\% and $9.7 \%$ respectively. A review of studies in developing and industrialized countries ${ }^{[4]}$ gave a median value of $32.0 \%$ among children and women of childbearing age while another in China [7] reported a rate of $37.8 \%$ in a retrospective study across a range of paediatric practise among children aged 3 to 18 months.

The study at Ilorin [8] attributed partial immunisation to long waiting times in the clinics, non-availability of vaccines, parent's objection and concerns for the safety of vaccines in $15.2 \%, 17.5 \%$ and $38.8 \%$ of cases respectively. Similarly, a study in Mozambique by Jani et al. [12] attributed partial immunisation to health service issues in $38.3 \%$, and poor access to facilities in $15.6 \%$ of cases. The review of studies [4] found that institutional challenges such as logistic problems were implicated in $10 \%$ of cases while failure to administer vaccines simultaneously, fear of wastage of vaccines, the institution of false contraindications and negative attitude of health workers accounted for $\mathrm{MOV}$ in $22 \%, 17 \%, 18 \%$ and $16 \%$ of cases respectively.

Investigating $\mathrm{MOV}$ in the first year of life is important as it enables such susceptible children to be identified early in life, for the optimisation of their immunisation status. Immunisation is carried out most frequently in primary health care centres in Lagos State. Nevertheless, a significant number of children present to this centre, the Lagos State University Teaching Hospital (LASUTH) which has highly qualified 
cadre of personnel for high-quality care, including routine immunisation services. Therefore, it is necessary to evaluate their immunisation status to ensure that they are adequately immunised at discharge and during follow-up care. This study was conducted to determine the prevalence of missed opportunities for vaccination and the factors associated with it among children seen at the General Outpatient and Newborn follow up clinics at the Lagos State University Teaching Hospital Ikeja (LASUTH).

\section{Methods}

\section{Study design}

A health facility-based descriptive, crosssectional study was carried out between March 1, and August 31, 2016.

\section{Study settings}

The study site was the Newborn clinic and General Outpatient clinics at the Lagos State University Teaching Hospital, Ikeja, Lagos in southwest Nigeria. It is one of the four tertiary hospitals in Lagos. The paediatric section of the hospital offers emergency, general outpatient services and specialist services. The Newborn clinic caters for babies admitted and discharged from the newborn unit up to the age of one year. It runs weekly and attends to an average of forty babies a week. The General Outpatient clinic attends to all paediatric patients presenting with minor ailments outside of specific clinic days and runs every day of the week except weekends. It attends to two hundred infants monthly.

\section{Study population}

The study population included caregivers attending the new-born follow up and general out-patient clinics whose children were aged 0 12 months.

\section{Sample size determination}

The minimum sample size for the study was determined using the appropriate formula for cross-sectional studies. [15] The statistical assumptions included prevalence of $\mathrm{MOV}$ of $33 \%,[8]$ a type 1 error of $5 \%$, precision of $6 \%$ and a non-response rate of $10 \%$, thus arriving at a minimum sample size of 256 children.

Sampling techniques

Mothers and caregivers who attended the clinics were consecutively recruited at the point of exit after obtaining informed consent.

\section{Study instrument and data collection}

Using a structured questionnaire, five trained resident doctors in the Department of Paediatrics collected data on the demographic characteristics of the children and their caregivers. The parental socioeconomic status was determined using the scheme recommended by Oyedeji. [16] This scheme grades socio-economic status into three classes the upper (classes I and II), middle (class III) and lower (classes IV and V). The knowledge of the number of vaccines which ought to have been received and previous complications of vaccination were recorded. The history of prior contact with another health facility and attempts to update vaccinations in that facility were also obtained. The immunisation records were requested for (where available) and evaluated to determine the immunisation status and to verify claims in addition to verbal reports.

For children who did not complete routine immunisation, the reasons for this recorded and efforts were made to determine the presence of contraindications to immunisation.

Definition of missed opportunity: In this study, a child is said to have missed opportunity for vaccination when he or she is eligible by virtue of age, has no contraindications to vaccination, visits a health facility and fails to receive all the vaccines he or she is due for.

Contraindications to immunisation: Acute illness necessitating admission and high-grade fever.

Definition of vaccination statuses: 
1-Immunised up to date - children who were fully vaccinated (FV) and those partially vaccinated but up to date.

A - Fully vaccinated - children who were old enough to receive all the routines vaccines and had received all such vaccines in the NPI schedule at the exit.

B - Partially vaccinated up to date - a child had received all vaccines appropriate for the age in the NPI schedule at the exit but the child was not old enough to receive all vaccines.

2- Partially immunised not up to date - children who were not fully vaccinated and have not received all vaccines appropriate for age at the exit from the facility. These included two groups of children:

A- Those who were not fully immunised and had contraindications (C.I) to vaccination.

B - Those who were not fully immunized and had no contraindications (no C.I) to vaccination. These were also regarded to have Missed Opportunity for Vaccination (MOV).

3 -Unvaccinated - had received no vaccines.

\section{Ethical considerations}

Ethical approval for the study was obtained with - Ethics Clearance Certificate number LREC/06/10/686.

\section{Data analysis}

Data were extracted, imputed and analysed using the Statistical Package for Social Sciences version 22.0. Percentages of categorical variables were determined. The prevalence of MOV was calculated. Chi-Square test was used to compare two independent categorical variables. $P$ values less than 0.05 were considered statistically significant.

\section{Results}

Two hundred and fifty-six children were studied. There were 149 (58.2\%) males and 64 $(22.9 \%)$ neonates, with $4(1.6 \%)$ in the early neonatal period. The mean age was $17.9 \pm 15.9$ weeks. The duration of gestation ranged between 28 and 44 weeks with $83.6 \%$ delivered full-term (with a mean of 38.7 weeks) and $16.4 \%$ delivered as preterm. Two hundred and eleven $(82.4 \%)$ of the deliveries took place at health facilities. Analysis of the birth order revealed that $83.6 \%$ were the first four pregnancies with a range of 1 to 6 and a mean of 1.93. More than three quarters $(82.8 \%)$ of the caregivers were at least twenty years old but not more than thirtyfive years with the mean age of $31.1 \pm 4.7$ years. Nearly three quarters $(73.4 \%)$ were of the Christian faith. Educational attainments were comparable among the fathers and mothers with tertiary education in $68.0 \%$ and $61.3 \%$ respectively. Further, $79.3 \%$ and $9.8 \%$ of the families belonged to the upper and lower socioeconomic classes respectively as shown in Table I

The pattern of vaccination coverage and status revealed the following: the earlier vaccines ( $\mathrm{BCG}, \mathrm{OPV}_{0}$ and $\mathrm{HBV}_{0}$ ) had the highest coverage with rates of $89.1 \%, 89.1 \%$ and $88.3 \%$ respectively. This is followed by the three Pentavalent/Oral Polio Vaccine pairs (Pentavalent $1 / \mathrm{OPV}_{1}$, Pentavalent $2 / \mathrm{OPV}_{2}$ and Pentavalent $\mathrm{O}_{3} / \mathrm{OPV}_{3}$ ) with rates of $64.1 \% / 64.8 \%$, $50.0 \% / 50.4 \%$, and $41.4 \% / 41.8 \%$ respectively. The vaccines administered to the older children had the lowest coverage $16.4 \%$ and $14.5 \%$ for measles and Yellow fever vaccines.

Table II shows that thirty-six (14.1\%) of the subjects were fully vaccinated. However, 177 $(69.1 \%)$ of the remaining subjects who were not fully vaccinated were up to date with their vaccines while $16(6.2 \%)$ were not eligible for vaccination as they had valid contraindications. Following the exclusion of the aforementioned 16 children with genuine contraindications to vaccination, 27 of the remaining 240 eligible children were not vaccinated giving an overall 
prevalence of $11.3 \%$ for missed opportunities for vaccination.

Bivariate analyses of factors associated with MOV rates revealed significant statistical associations with gender $(\mathrm{p}=0.04)$ and parental socio-economic status $(\mathrm{p}=0.008)$ (Table III).
Only $44(18.3 \%)$ of the 240 children who were eligible but not fully vaccinated were indeed evaluated by health workers for their immunisation status.

Table I: Socio-demographic characteristics of the 256 children studied

\begin{tabular}{|c|c|c|}
\hline Characteristics & Frequency & Percentage \\
\hline \multicolumn{3}{|l|}{ Age (weeks) } \\
\hline$\leq 4$ & 64 & 25.0 \\
\hline$>4-52$ & 192 & 75.0 \\
\hline Mean $\pm S D$ & $17.9 \pm 15.93$ & \\
\hline \multicolumn{3}{|l|}{ Gender } \\
\hline Female & 107 & 41.8 \\
\hline Male & 149 & 58.2 \\
\hline \multicolumn{3}{|l|}{ Place of delivery } \\
\hline Hospital & 211 & 82.4 \\
\hline Non-hospital & 45 & 17.6 \\
\hline \multicolumn{3}{|l|}{ Birth order } \\
\hline$<4$ & 214 & 83.6 \\
\hline$\geq 4$ & 42 & 16.4 \\
\hline \multicolumn{3}{|l|}{ Age of caregivers (years) } \\
\hline$<20$ & 2 & 0.8 \\
\hline $20-34$ & 210 & 82.0 \\
\hline$>35$ & 44 & 17.2 \\
\hline \multicolumn{3}{|l|}{ Religion } \\
\hline Christianity & 188 & 73.4 \\
\hline Islam & 68 & 26.6 \\
\hline \multicolumn{3}{|l|}{ Paternal Educational status } \\
\hline University graduates or equivalents & 174 & 68.0 \\
\hline $\begin{array}{l}\text { School certificate, Ordinary level (GCE) with teaching/ } \\
\text { professional training }\end{array}$ & 64 & 25.0 \\
\hline School certificate or Grade II teachers certificate or equivalents & 15 & 5.9 \\
\hline Modern three and primary six certificate & 2 & 0.7 \\
\hline Those who could either just read or write or were illiterates & 1 & 0.3 \\
\hline \multicolumn{3}{|l|}{ Maternal Educational status } \\
\hline University graduates or equivalents & 157 & 61.3 \\
\hline School certificate, Ordinary level (GCE) with teaching/ & 79 & 30.9 \\
\hline professional training & 15 & 5.9 \\
\hline \multicolumn{3}{|l|}{ School certificate or Grade II teachers certificate or equivalents } \\
\hline Modern three and primary six certificate & 3 & 1.2 \\
\hline Those who could either just read or write or were illiterates & 2 & 0.9 \\
\hline \multicolumn{3}{|l|}{ Parental socioeconomic status } \\
\hline Upper & 203 & 79.3 \\
\hline Middle & 29 & 11.3 \\
\hline Lower & 24 & 9.4 \\
\hline
\end{tabular}

The individual prevalence rates of MOV among females, males, preterm infants, term infants, neonatal and post-neonatal children were respectively. Further, the proportions of these children with MOV concerning the gender, maturity and chronological age revealed a preponderance of males $(77.8 \%)$, term delivery $(88.9 \%)$ and post-neonatal age $(63.0 \%)$ 
respectively. Children who were products of hospital-based deliveries (77.8\%) and whose parents were in the upper socio-economic class $(51.8 \%)$ had higher prevalence rates of MOV.

Table IV shows the various reasons for MOV but the responses were multiple. These included non- availability of the required vaccines in 15
(55.5\%), reluctance to administer more than one vaccine that the child is due for in 14 (51.8\%), failure to get the right number of children to receive vaccines to avoid wastage in $5(18.5 \%)$ cases respectively. The failure of the guardian to give consent and previous complications of immunisation were reasons for MOV in $2(7.4 \%)$ cases.

Table II: Total coverage for each vaccine and vaccination status of the children studied

\begin{tabular}{lll}
\hline Vaccine & Coverage (\%) & Missed doses \\
\hline BCG & 89.1 & 17 \\
$\mathrm{OPV}_{0}$ & 89.1 & 15 \\
$\mathrm{HBV}_{0}$ & 88.3 & 17 \\
Pentavalent $_{1}$ & 64.1 & 8 \\
$\mathrm{OPV}_{1}$ & 64.8 & 8 \\
Pentavalent & \\
OPV $_{3}$ & 50.0 & 5 \\
Pentavalent & 50.4 & 5 \\
OPV & 41.4 & 4 \\
Measles & 41.8 & 4 \\
Yellow Fever & 16.4 & 5 \\
& 14.5 & 1 \\
Vaccination status & & Percentage \\
Fully vaccinated & Frequency & 14.1 \\
Not fully vaccinated: vaccinated up to date & $36 / 256$ & 69.1 \\
Not fully vaccinated: not vaccinated up to date but & $27 / 240$ & 11.3 \\
with no contraindication (missed opportunities) & & \\
Not fully vaccinated: vaccinated up to date with & $16 / 256$ & 6.3 \\
genuine contraindications & & \\
\hline
\end{tabular}

\section{Discussion}

The prevalence of MOV in this study was $11.3 \%$. This is lower than the rate obtained in two different studies in Ilorin [8,9] and Calabar, [10] among children under one year and under five years of age respectively. It is also lower than the rate reported from Sudan [5] but comparable to the rate obtained from Nnewi [11] and Argentina. [13] The study findings might reflect the larger size and the fact that the subjects had been resident in the area of study for over 18 months in the latter study. However, a study among children aged up to 59 months in different health facilities across Cape Town, South Africa [17] yielded a much lower MOV rate. This may reflect better health services in these facilities as children who came for immunisation were excluded from the study. The implication of the prevalence of MOV in this study is that one out of nine children remained unimmunized and are, therefore, susceptible to vaccine-preventable diseases (VPD). Individual prevalence rates showed a preponderance of MOV among the male, term deliveries, post-neonatal age as well as those with parents from the upper and middle socioeconomic classes. This is at variance with 
the assertion from a study in Brazil [18] and in a study in Gusau, northern Nigeria [19] which found that MOV was higher among children from parents with lower socioeconomic status.

Table III: Association between respondents' characteristics and frequency of Missed Opportunity for Vaccination

\begin{tabular}{|c|c|c|c|c|c|}
\hline \multirow[t]{4}{*}{ Characteristics } & & \multicolumn{2}{|c|}{ Missed opportunities } & \multirow[t]{4}{*}{ Total } & \multirow[t]{4}{*}{$p$-value } \\
\hline & & Yes & No & & \\
\hline & & $\mathrm{n}=27$ & $\mathrm{n}=213$ & & \\
\hline & & $(\%)$ & $(\%)$ & & \\
\hline \multicolumn{6}{|l|}{ Gender } \\
\hline & Female & $6(6.1)$ & $92(93.9)$ & 98 & 0.04 \\
\hline & Male & $21(14.8)$ & $121(85.2)$ & 142 & \\
\hline \multicolumn{6}{|l|}{ Maturity } \\
\hline & Preterm & $3(8.8)$ & $31(91.2)$ & 34 & 0.63 \\
\hline & Term & $24(11.7)$ & $182(88.3)$ & 206 & \\
\hline \multicolumn{6}{|l|}{ Age (weeks) } \\
\hline & $\leq 4$ & $10(18.2)$ & $45(81.8)$ & 55 & 0.064 \\
\hline & $>4-52$ & $17(9.2)$ & $168(90.8)$ & 185 & \\
\hline \multicolumn{6}{|l|}{ Place of delivery } \\
\hline & Hospital & $21(10.6)$ & $177(89.4)$ & 198 & 0.49 \\
\hline & Non-hospital & $6(14.3)$ & $36(85.7)$ & 42 & \\
\hline \multicolumn{6}{|l|}{ Parental SES } \\
\hline & Upper & $14(7.9)$ & $163(92.1)$ & 177 & 0.008 \\
\hline & Middle & $12(23.5)$ & $39(76.5)$ & 51 & \\
\hline & Lower & $1(8.3)$ & $11(91.7)$ & 12 & \\
\hline
\end{tabular}

Table IV: Reasons for Missed Vaccination Opportunities

\begin{tabular}{lll}
\hline Reasons & Frequency & Percentage \\
\hline Non-availability of required vaccines & 15 & 55.5 \\
Reluctance to administer more than one vaccine & 14 & 51.8 \\
$\begin{array}{l}\text { Failure to gather the required number of subjects to } \\
\text { exhaust a vial }\end{array}$ & 5 & 18.5 \\
$\begin{array}{l}\text { Failure of guardian consent } \\
\text { Previous complications }\end{array}$ & 1 & 3.7 \\
\hline
\end{tabular}

This is likely because of poor understanding of the importance of the benefits of immunisation among this group of individuals. The higher MOV rates in the middle and upper socioeconomic classes may be a consequence of the high percentage of the subjects in the upper socio-economic class signifying that this cadre of caregivers is more likely to walk in and seek care or seek a referral to specialist centres for care. The preponderance of MOV noted among male subjects is interesting as it is expected that culturally, male children are given health preferences than females. It may also arise from the fact that more males are likely to be brought to the health facility for care. However, the study from Gusau [19] noted that more males 
were likely to be fully immunised than females. The observation of term delivery and postneonatal age accounting for a significant proportion of MOV in the present study may be as a result of the high percentage compared to premature subjects. Many times, preterm subjects are more likely to have missed opportunities for vaccination due to the possibility of prolonged hospital stay on account of complications that ensue after birth.

A decrease in the appropriateness of vaccination coverage with increasing age of the subjects was observed. While the earliest vaccines ie BCG and the first oral polio vaccine $\left(\mathrm{OPV}_{0}\right)$ had high coverage, measles and Yellow fever vaccines had remarkably lower coverage rates. This is similar to what has been reported in studies in Sabongida-Ora in Edo State ${ }^{25}$ and in Sudan. [5] This usually occurs as a result of the long interval between the third dose of the pentavalent vaccines and yellow fever and measles vaccines.

The percentage of children who were fully immunized in the present study is lower in comparison to the finding from Mozambique, [12] which had a completion rate of $72.8 \%$ among children who were aged less than two years. Etuk in Calabar, [5] who studied older children also obtained a higher rate of full immunisation of $82.2 \%$. This is unusual because the older the children the lower the expected proportion with full immunisation. A majority of the children in the present study were up to date but not fully immunized.

The gender and parental socio-economic status were the only factors significantly associated with MOV. While reasons for more males experiencing MOV more significantly may not be readily known, that of the inverse relationship between parental socio-economic status and MOV $[5,8,10]$ is well known. This was reported in a study in Ilorin, [8] Sudan [5] and also in Calabar. [10] Therefore, it may be important to develop messages that will target parents in the lower-socio-economic classes.

Clinical review of the vaccination status of children by health care workers and subsequent referral for vaccination before exit happened in only $18.3 \%$ of eligible children. This may be as a result of the failure to continuously update the knowledge of health workers on the importance of this intervention in reducing MOV. Failure to make enquiries of the vaccination status of children who were seen in an outpatient clinic was found to be a risk factor for MOV in a high number of cases in a study in India [23] and a cause of $\mathrm{MOV}$ in $27 \%$ of patients who attended an outpatient facility in Seattle, USA. [6] A review of studies in small and medium scale countries in Africa [24] found that the percentage of health care workers who reviewed the immunisation status of children on a sick visit in for-profit and not-for-profit facilities ranged between $14 \%$ and $44 \%$. An intervention study which sought to train health workers to assess the immunisation status of children who visited the health facility on a sick visit and, immunisation and follow-up care visits showed a reduction in the prevalence of MOV. [14]

The reasons for MOV in the present study were multiple in some subjects. Non- availability of the required vaccines was noted in a large number of subjects. This was similar to what was reported in Nnewi, Nigeria [11] and a review of studies in some developing and industrialized countries. [4] The prevalence of such in the present study is higher than what was obtained in earlier studies. This might be a result of poor logistics and poor power supply which is prevalent in this environment, thus affecting the storage and stock of vaccines. The reluctance to administer more than one vaccine, that the child is due for at a time, is the next common reason for MOV. However, this is higher than the finding obtained in a review of studies [4] and a study in India. [12] This is probably linked to a 
fear of the possible complications of multiple vaccinations. Failure of guardians to give consent for vaccination and previous complications of vaccinations were reasons in a few cases. The former can occur if there is inadequate knowledge on the part of the caregiver about the benefits of immunisation and inadequate counselling by the health worker. Failure to get the right number of children required to exhaust vials of the vaccine and reluctance to open multi-dose vials of the vaccine to avoid wastage were also adduced to be responsible for MOV. This is similar to values obtained in a review study by Hutchins who classified this as a negative attitude amongst health workers and was responsible for MOV in almost a fifth of cases. It could be due to inadequate resources especially in public institutions where routine vaccination services are free.

It was also observed that some false contraindications to vaccination were adopted by health care workers, particularly the small body size of the child. Health workers are being discouraged from using this as a reason not to vaccinate children as these infants who may be products of preterm gestation or small for gestational age are as prone to vaccinepreventable diseases (VPDs) just like the bigger infants.

Reliance on verbal information from some of the caregivers in the absence of immunization records is acknowledged as a limitation to this study.

\section{Conclusion}

This study identified upper socioeconomic status and the male gender to be significantly associated with MOV. Messages and outreaches about the benefits of vaccination should be targeted at parents in the upper and middle socioeconomic groups through media houses and religious institutions. It was also noted that some health workers do not routinely assess the vaccination status of children at follow-up or sick child visits to detect children who are partially immunized but eligible for vaccination. It may be recommended that children, particularly in the first year of life, should have their immunisation status reviewed at every contact with health facilities and should be appropriately referred for vaccination in the absence of contraindications. Health workers should also be trained on the true contraindications to vaccination. This is to avoid instituting false contraindications and consequently improve vaccination rates in vulnerable children.

Authors' Contributions: KOA and OOO conceived and designed the research., KOA, KYA and $\mathrm{OOO}$ participated in data collection, data analysis and interpretation, and drafting of the manuscript. All the authors approved the final version of the manuscript.

Conflict of interest: None declared.

Funding: Self-funded.

Publication History: Submitted 01 March 2020; Accepted 16 May 2020.

\section{References}

1. Centre for Global Development. Making Markets for vaccines: from ideas to actions. Washington DC: Centre for Global Development; 2005.

2. World Health Organization. Immunization surveillance, assessment and monitoring. http://www.who.int/immunization_monit oring/diseases/en/

3. WHO/UNICEF. Global immunization vision and strategy, 2006-2015. Geneva, Switzerland and New York. 2005.

4. Hutchins SS, Jansen HA, Robertson SE, Evans P, Kim-Farley RJ. Studies of missed opportunities for immunization in 
developing and industrialized countries. Bull World Health Org 1993; 71: 549-550.

5. Malual AC, Jowi Y, Irimu G, Admani B. Missed opportunities for immunization among children attending a Paediatric Outpatient Clinic at Juba Teaching Hospital. South Sudan Med J 2018; 11: 36-39.

6. Watson MA, Feldman KW, Sugar NF, Sommer CJ, Thomas ER, Lin T. Inadequate history as a barrier to immunization. Arch Pediatr Adolesc Med 1996; 150:135-139.

7. Fu LY, Zook K, Gingold J, Gillespie CW, Briccetti C, Cora-Bramble D, et al. Frequent vaccination missed opportunities at primary care encounters contribute to under immunization. The J of Pediatr 2015; 166: 412-417.

8. Abdulraheem IS, Onajole AT, Jimoh AAG, Oladipo AR. Reasons for incomplete vaccination and factors for missed opportunities among rural Nigerian children. J Public Health Epidemiol2011; 3: 194-203.

9. Aderibigbe SA, Alatishe-Muhammad BW, Ameen HA, Bolarinwa OA, Salaudeen AG, Uthman MM, et al. Determinants of Missed Opportunities for Immunization among Under-five children in Ilorin Metropolis. Trop J Health Sci 2017; 24: 18-23.

10. Anah MU, Etuk IS, Udo JJ. Opportunistic immunization with in-patient programme: eliminating a missed opportunity in Calabar, Nigeria. Ann Afr Med 2006; 5: 188-191.

11. Ubajaka FC, Ukegbu AU, Okafor NJ, Ejiofor O. The prevalence of missed opportunities for immunization among children utilizing immunization services in Nnamdi Azikiwe University Teaching Hospital, Nnewi. J Biol Agric Health 2012; 6:112-119.

12. Jani JV, De Schacht C, Jani IV, Bjune G. Risk factors for incomplete vaccination and missed opportunity for immunization in rural Mozambique. BMC Public Health 2008; 8: 161.

13. Gentile A, Bakir J, Firpo V, Caruso M, Lución MF, Abate HJ, et al. Delayed vaccine schedule and missed opportunities for vaccination in children up to 24 months. A multicentre study. Archivos Argentinos de Pediatria 2011; 109: 219-225.

14. Deivanayagam N, Nedunchelian K, Mala N, Ashok TP, Rathnam SR, Ahmed SS. Missed opportunities for immunization in children under 2 year attending an urban teaching hospital. Indian Pediatr 1995; 32: 51-57.

15. Pourhoseingholi MA, Vahedi M, Rahimzadeh M. Sample size calculation in medical studies. Gastroenterol Hepatol 2013; 6: 14-17.

16. Oyedeji GA.Socioeconomic and cultural background of hospitalized children in Ilesa. Niger J Paediatr 1985; 12: 111-117.

17. Jacob N, Coetzee D. Missed opportunities for immunisation in health facilities in Cape Town, South Africa. South Afr Med J 2015; 105: 917-921.

18. Restrepo-Méndez MC, Barros AJ, Wong KL, Johnson HI, Pariyo G, Wehrmeister FC, et al. Missed opportunities in full immunization coverage: findings from low-and lowermiddle-income countries. Global Health Action 2016; 9: 30963.

19. Ilah BG, Sakajiki AM, Musa A, Edem B, Adelakun $\mathrm{MB}$, Adenji $\mathrm{AO}$, et al. Immunisation status of children 12-59 months attending a specialist hospital, Gusau, Nigeria. Ann Trop Med Public Health 2015; 8: 23-27.

20. Ndwandwe D, Uthman OA, Adamu AA, Sa mbala EZ, Wiyeh AB, Olukade T, et al. Decomposing the gap in missed opportunities for vaccination between poor and non-poor in sub-Saharan Africa: a multicountry analysis. Hum Vaccine 
Immunother 2018; $\quad$ 14: $\quad$ 2358-2364. doi:10.1080/21645515.2018.1467685.

21. World Health Organization. Immunization surveillance, assessment and monitoring. Vaccine-preventable diseases. 2012. http://www.who.int/immunization_monit oring/diseases/en/. Accessed 2 July 2019.

22. World Health Organization. Missed Opportunities for Vaccination (MOV) Strategy 2017. Available from: http://www.who.int/immunization/progra mmes_systems/policies_strategies/MOV/e n/. Accessed 2 July 2019.

23. Verma SK, Mourya HK, Yadav A, Mourya S, Dabi DR. Assessment of missed opportunities of immunization in children visiting a health facility. Int J Contemp Pediatr 2017; 4: 1748-1753.

24. Olorunsaiye CZ, Langhamer MS, Wallace AS , Watkins ML. Missed opportunities and barriers for vaccination: a descriptive analysis of private and public health facilities in four African countries. Pan Afr Med J 2017; 27(Suppl 3): 6. doi:10.11604/pamj.supp.2017.27.3.12083.

25. Odusanya OO, Alufohai EF, Meurice FP, Ahonkhai VI. Determinants of vaccination coverage in rural Nigeria. BMC Public Health 2008; 8: 381. doi:10.1186/1471-2458-8381. 